\title{
Panton-Valentine Leukocidin producing Staphylococcus aureus facial pyomyositis causing partial cavernous sinus thrombosis
}

Katherine Green MBBS ${ }^{\star 1}$, Ioanna Chranioti MBChB ${ }^{\star 2}$, Saurabh Singh MBBChir ${ }^{3}$, Hans Rolf Jäger $\mathrm{FRCR}^{3,4}$, Anje Drebes MRCP, FRCPath ${ }^{5}$, Susie Gabbie MRCPCH, MSc ${ }^{1}$ and Jonathan Cohen MRCPCH PhD \pm 2

${ }^{1}$ Department of Paediatrics, Royal Free London NHS Foundation Trust, UK; ${ }^{2}$ Paediatric \& Adolescent Division, University College Hospitals NHS Foundation Trust, UK, ${ }^{3}$ Centre of Medical Imaging, University College London, ${ }^{4} \mathrm{UCL}$ Institute of Neurology Academic Neuroradiological Unit, University College London, UK; ${ }^{5}$ KD Haemophilia and Thrombosis Centre, Royal Free London NHS Foundation Trust, UK

*The first two authors contributed equally to this manuscript

\pm Corresponding author:

Paediatric \& Adolescent Division

University College London Hospitals NHS Foundation Trust

$6^{\text {th }}$ Floor Central

250 Euston Road

London

NW1 2PG

Phone: +44 2034477876 Fax: +44 2034479064

E-mail: jonathan.cohen@uclh.nhs.uk

Abbreviated Title: Facial pyomyositis causing cavernous sinus thrombosis

Running Title: Pyomyositis causing cavernous sinus thrombosis

Keywords: Panton-Valentine leukocidin; Staphylococcus aureus ; pyomyositis; cavernous sinus thrombosis 


\section{Abstract}

We present a case of sub-total cavernous sinus thrombosis secondary to Panton-Valentine leucocidin-associated Staphylococcus aureus pyomyositis of the muscles of mastication in a previously healthy child, who was successfully managed with no residual disease. He was found to have a factor $V$ Leiden heterozygous mutation. We highlight the propensity of PVLSA to induce venous thrombosis at any site, but with potential for more severe consequences in the head. We raise attention to pyomyositis as a differential for peri-orbital cellulltitis, and also discuss the significance of the factor $\mathrm{V}$ Leiden mutation. 


\section{Case Report}

A 13-year-old white British male presented with a 5-day history of headaches, malaise and unilateral left sided facial swelling followed by pyrexia. He also reported pain on mastication and blurring of left sided vision and his mother reported vague episodes of confusion. He had been prescribed co-amoxiclav, ibuprofen and paracetamol by his GP with no clinical improvement. There was no history of trauma to the area. There was a past medical history of mild allergic rhinitis but he took no regular medications. There was no family history of immunodeficiency. On examination the patient was pyrexial with extensive facial and orbital tenderness and erythematous swelling, extending from the left forehead to the left temporomandibular joint. Neurological and visual examinations were normal. The left tympanic membrane was bulging but without signs of perforation. Cardiovascular, respiratory and abdominal examinations were normal. External ocular movements were normal.

At presentation, the C-reactive protein was $278 \mathrm{mg} / \mathrm{L}$. Full blood count was normal with white count $6.28 \times 10^{9} / \mathrm{L}$, neutrophils $5.07 \times 10^{9} / \mathrm{L}$ and platelet count $182 \times 10^{9} / \mathrm{L}$. A clinical diagnosis of peri-orbital cellulitis was made. The significance of the unusual symptoms of pain on mastication was not initially noted by the treating clinicians. Intravenous ceftriaxone and metronidazole were commenced. After forty-eight hours there was no clinical improvement. The patient underwent enhanced CT imaging of the head, facial bones and orbit. The initial imaging findings included opacification of the sphenoid sinus with an airfluid level but no bony erosion. In addition there was an expanded left cavernous sinus containing a filling defect associated with a prominent left superior ophthalmic vein in keeping with partial left cavernous sinus thrombosis (Figure 1A). The patient was transferred to the local tertiary centre for further management for consideration of drainage of the sphenoid collection. Opinion from otorhinolaryngology was that the sphenoid disease was not significant enough to be the source of infection and re-consideration of the CT 
images identified diffuse thickening of the left temporalis, masseter and pterygoid muscles (Figure 1B) with some mild enhancement with formation of small collection around the superior and medial aspect of the pterygoid muscles, too small to drain. The significance of the pain on mastication and swelling extending to the tempero-mandibular joint was then appreciated to be due to facial soft tissue infection. The concurrent rhinosinusitis was considered minor and not the source of infection. Methicillin-sensitive Staphylococcal aureus (MSSA) had been cultured from the blood at the original hospital but blood cultures were sterile subsequently by the time of transfer. Due to response and ease of administration, a decision was made to continue ceftriaxone with additional clindamycin in place of metronidazole. It was considered that the source of infection was pyomyositis of the facial muscles. Levels of serum immunoglobulins and nitroblue tetrazolium testing were all normal. HIV test was negative. Echocardiogram was performed and did not demonstrate any cardiac vegetations. Anticoagulation was initially commenced with dalteparin prior to starting warfarin therapy.

The child improved clinically with intravenous ceftriaxone and oral clarithromycin. The MSSA was typed as Panton-Valentine leucocidin (PVL) positive. In view of the good therapeutic response, antibiotics were not changed. He was discharged home for ongoing antibiotic and anticoagulant therapy in the community.

Repeat enhanced MR imaging after two months showed significant improvement of muscle swelling, decrease in size of the collection but incomplete resolution of the cavernous sinus thrombosis. Ceftriaxone had been stopped due to development of a rash attributed to allergy to ceftriaxone. In view of incomplete resolution of the thrombosis and the potential for viable bacteria to persist within it, clindamycin was continued for a further two months until a further MRI demonstrated resolution of thrombosis and resolution of the swelling of the muscles of mastication (Figure 1C-D). The patient and household members all underwent topical and nasal Staphylococcus aureus decolonisation therapy. Anti-coagulation was continued for a 
total of six months and followed by a thrombophilia screen. The only abnormality found was that he had a heterozygous factor $\mathrm{V}$ Leiden mutation.

\section{Discussion}

Pyomyositis is an acute bacterial infection of skeletal muscle with localized abscess formation. Staphylococcus aureus (SA) accounts for $75 \%-90 \%$ of these infections (ref Verma 2016). Primary pyomyositis is a rare disease, as skeletal muscles are relatively resistant to bacterial infections. However, under certain circumstances, $S$. aureus can invade muscle groups via haematogenous spread. This usually occurs without evidence of spread from a contiguous structure or through a cutaneous portal of entry.

Although several case studies of pyomyositis in children and adults have been published, many of the patients described in the earlier literature had underlying conditions, such as immunodeficiency or diabetes mellitus (ref Pannaraj CID 2006). Pyomyositis typically occurs in the absence of direct percutaneous inoculation, and is believed to arise through haematogenous spread, often amongst those engaged in more exercise and at sites of previous minor trauma such as such as those around the hips (ref Unnikrishna Int Orthop 2010). , often several days prior to infection becoming apparent. (ref Verma). In the last 15 years, reports of SA pyomyositis in temperate climates have increased (ref Unnikrishna). Many of these are associated with the production of PVL (PVL-SA) (4). Whilst there have been occasional reports of PVL-SA causing facial cellulitis or cervical lymphadenitis, to our knowledge, this is the first report of PVL-SA pyomyositis affecting the face. Due to its rarity, facial soft tissue infection was not considered by the treating clinicians, despite the history of 
pain on mastication and lateral facial swelling which are not typically seen in peri-orbital or orbital cellulitis. There is a lack of evidence to guide management decisions in PVL-SA (ref Ritz PIDJ 2012). Expert opinion has suggested use of clindamycin and rifampicin to switch of toxin production in PVL-SA, such as in UK guidance (ref HPA 2008). In the case presented here, the patient responded quickly to clindamycin alongside ceftriaxone. It is theoretically possible that use of rifampicin or linezolid in addition may have led to more rapid resolution. He received a prolonged treatment course in case of viable bacteria surviving within the endovascular thrombosis. Antimicrobial therapy was stopped once the thrombosis had fully resolved.

Paediatric cavernous sinus thrombosis (CST) is a rare and life-threatening complication of septic or aseptic etiologies, and is associated with low morbidity and mortality if aggressive therapies including antimicrobials, anticoagulation, and/or surgical treatment are implemented early on. CST is associated with neurologic disability if not detected in a timely manner. Classic presentations of CST include abrupt onset of unilateral periorbital oedema, headache, photophobia and proptosis. Although, our patient did not have clinical signs specific to CST, there was enlargement of the left ophthalmic vein accompanied by a filling defect, evidence of a partial occlusion. Without swift therapy it may have progressed to total occlusion.

There is a greater propensity for $S$. aureus infections caused by PVL-producing strains to be complicated by deep vein thrombosis (DVT). Facial SA infection is a recognized risk factor for CST (ref Desa 2012), with PVL-SA increasing the risk further. There is general consensus that anti-coagulation therapy should be given for DVT in PVL-SA infection (ref Ritz PIDJ 2012), with the risk of haemorrhage being outweighed by the benefit of resolving current thrombosis and limiting the development of further thrombosis when the endothelium and platelets are activated. For cerebral venous thrombosis (CVT), however, the consequences of haemorrhage are highly significant. Whilst studies are limited and not randomized in children, both European (ref Lebas 2012) and British guidelines (ref BSCH) 
recommend anti-coagulation with $\mathrm{LMWH}$ and then vitamin $\mathrm{K}$ antagonists for CVT in adults. Both guidelines recommend 3 months duration for a transient provocation and six months for idiopathic. The British guideline also recommends this for children, although the European guideline is more circumspect stating such therapy as reasonable according to individual circumstances. Given the age of our patient, LMWH was initially commenced, and then switched to warfarin. Resolution of thrombosis took four months, so a total of six months anticoagulation was given in this case.

It is not clear whether the heterozygous factor $\mathrm{V}$ Leiden mutation in this patient made a significant contribution to thrombosis risk. Such mutations are common, present in $2-8 \%$ of Caucasian population (ref: $x x x$ ). Individuals with this mutation usually don't tend to present with venous thrombosis until they are adults. To our knowledge, there is only a single previous report of PVL-SA infection causing DVT in association with the heterozygous factor $\mathrm{V}$ Leiden state, but that patient also had a mutation in the prothrombin gene (ref: $A n$ uncommon presentation for a severe invasive infection due to methicillin-resistant Staphylococcus aureus clone USA300 in Italy: a case report. Piero Valentini, ${ }^{1}$ Gabriella Parisi, ${ }^{2}$ Monica Monaco, ${ }^{3}$ Francesca Crea, ${ }^{1}$ Teresa Spanu, ${ }^{4}$ Orazio Ranno, ${ }^{1}$ Mirella Tronci, ${ }^{2}$ and Annalisa Pantost ${ }^{3}$. We are not aware of studies that have explored whether this common mutation actually does increase the risk of DVT risk in PVL-SA infection.

We have presented a case of sub-total CST following PVL-SA pyomyositis of the left lateral pterygoid muscle in a healthy child, who was successfully managed with no residual disease. We highlight the propensity of PVL-SA to induce venous thrombosis at any site, but with potential for more severe consequences in the head. In cases of facial infection, we would strongly advocate close examination of imaging to look for evidence of even sub-total cavernous sinus occlusion, given the seriousness of the consequences.

\section{References}


1. Pannaraj P, Hulten K, Gonzalez B, Mason E and Kaplan S. Infective Pyomyositis and Myositis in Children in the Era of Community-Aquired, Methicillin-Resistan Staphylococcus aureus Infection. Clinical Infectious Disease. 2006;43:953-60.

2. Savvidou S, Kalogiannis E, Tsakiri K, Gavra M, Tsona A. Primary pyomyositis and disseminated septic pulmonary emboli: a reactivated staphylococcal infection? Braz $J$ Infect Dis. 2014;18(4):457-461.

3. Cunnington A, Brick T, Cooper M, Danin J, Hunt D, Jeanes A, Kearns AM, Nolan M, Lyall H. Severe invasive Panton-Valentine Leucocidin positive Staphylococcus aureus infections in children in London, UK. Journal of Infection. 2009;59:28-36.

4. Holmes A, Ganner M, McGuane, Pitt TL, Cookson BD and Kearns AM. Staphylococcus aureus Isolates Carrying Panton-Valentine Leucocidin Genes in England and Wales: Frequency, Characterization, and Association with Clinical Disease. Journal of Clinical Microbiology. 2005;43(5):2384-2390.

5. Unnikrishnan PN, Perry DC, George H, Bassi R and Bruce CE. Tropical primary pyomyositis in children of the UK: an emerging medical challenge. International Orthopaedics. 2010;34:109-113.

6. Health Protection Agency. Guidance on the diagnosis and management of PVLassociated Staphylococcus aureus infections (PVL-SA) in England. $2^{\text {nd }}$ Edition: 7 November 2008.

7. Smith DM, Vossough A, Vorona GA, Beslow LA, Ichord RN and Licht DJ. Pediatric cavernous sinus thrombosis: A case series and review of the literature. Neurology. 2015;85:763-769.

8. Elbright JR, Pace MT and Niazi AF. Septic Thrombosis of the Cavernous Sinuses. Arch Intern Med. 2001;161(22):2671-2676.

9. Desa V and Green R. Cavernous Sinus Thrombosis: Current Therapy. J Oral Maxillofac Surg. 2012;70:2085-2091.

10. Lyons C. The Treatment of Staphylococcal Cavernous Sinus Thrombophlebitis with Heparin and Chemotherapy. Annals of Surgery. 1941;113(1):113-117.

11. Einhäupl K, Stam J, Bousser MG, De Bruijn SFTM, Ferro JM, Martinelli I and Masuhr F. EFNS guidelines on the treatment of cerebral venous and sinus thrombosis in adult patients. European Journal of Neurology. 2010;17:1229-1235.

12. Lebas A, Chabrier S, Fluss J, Gordon K, Kossorotoff M, Nowak-Göttl U, De Vries LS and Tardieu M. EPNS/SFNP guidelines on the anticoagulant treatment of cerebral sinovenous thrombosis in children and neonates. European Journal of Paediatric Neurology. 2012;16:219-228.

13. Coutinho JM, Ferro JM, Canhão p, Barinagarrementeria F, Bousser M and Stam J. Unfractionated or Low-Molecular Weight Heparin for the Treatment of Cerebral Venous Thrombosis. Stroke. 2010;41:2575-2580. 


\section{Figure Legend}

Figure 1. (A) Enhanced venous phase CT image showing an expanded left cavernous sinus with a convex outer border containing a filling defect (arrow) at presentation. (B) Unenhanced coronal CT image showing diffuse swelling of the left temporalis muscle and marked asymmetry compared to the contralateral side (arrows) at presentation. (C) Enhanced T1-weighted image demonstrating normal enhancement of the cavernous sinus with no filling defects (arrow), four months after presentation. (D) Unenhanced T1-weighted, coronal image showing resolution of the left temporalis muscle swelling (arrows), four months after presentation. 


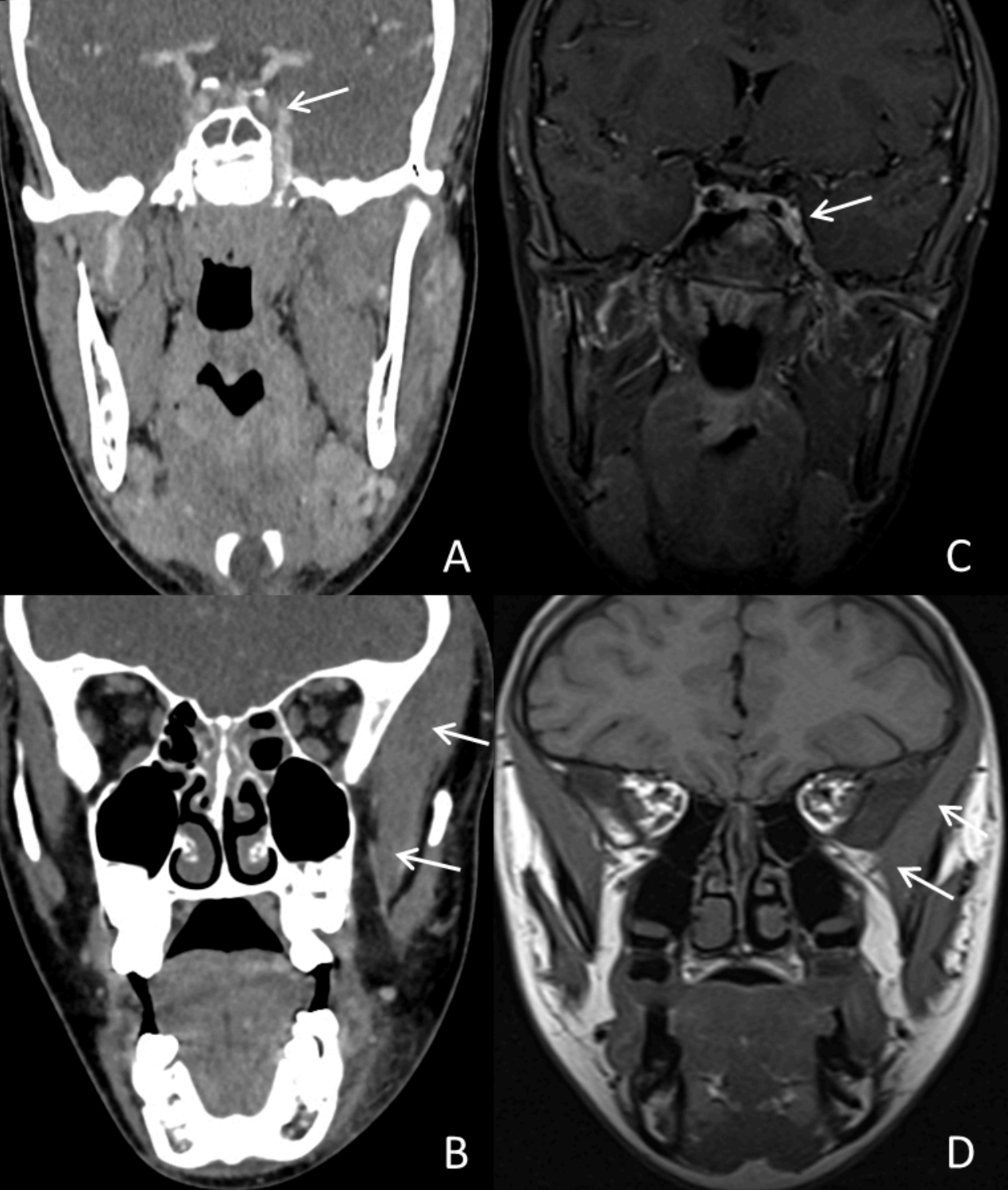

